\title{
Identification of developing multiple organ failure in sepsis patients with low or moderate SOFA scores
}

\author{
Gunnar Elke ${ }^{1 *}$ (D), Frank Bloos ${ }^{2,3}$, Darius Cameron Wilson ${ }^{4}$, Patrick Meybohm ${ }^{5}$ and the SepNet Critical Care Trials \\ Group
}

An early identification of sepsis patients likely to progress towards multiple organ failure is crucial in order to initiate targeted therapeutic strategies to decrease mortality. Our recent publication highlighted the greater accuracy of mid-regional proadrenomedullin (MR-proADM) compared with conventional biomarkers and clinical scores in predicting 28-day mortality in patients with initially low ( $\leq 7$ points; $N=240)$ or moderate $(8-13$ points; $N=653$ ) Sepsis-related Organ Failure Assessment (SOFA) scores [1], thus confirming results from smaller investigations $[2,3]$. This additional post hoc analysis aimed to further describe the non-surviving patient population of both subgroups and identify those likely to progress towards sepsis-related multiple organ failure.

In our study, patients with low SOFA scores had a lower 28 -day mortality rate $(N=35 ; 14.6 \%$ vs. $N=$ $181 ; 27.7 \%)$ and incidence of septic shock [4] $(N=$ $87 ; 36.7 \%$ vs. $N=399 ; 61.5 \%)$ compared to those with moderate values. Nevertheless, multiple organ failure was the most common cause of death irrespective of initial SOFA classification (low vs. moderate SOFA: $N=16 ; 45.7 \%$ vs. $N=79 ; 43.6 \%$ ). Patients with low SOFA scores tended to take longer to progress towards multiple organ failure (10 [6-18] vs. 7 [3-11] days) and had an increasing number of dysfunctional organs (identified by organ-specific SOFA scores $\geq 2$ ) and an increasing overall SOFA score (e.g. diagnosis to day 7: 2 [1-2] vs. 4 [3-5] dysfunctional organs; $P<0.01 ; 6.3 \pm 1.3$ vs. $10.2 \pm 4.7$ points; $P<0.01$ ).

Area under the receiver operating characteristic curve (AUROC) and Cox regression analysis indicated that MR-proADM had the highest accuracy in predicting progression towards sepsis-related multiple organ failure mortality in both groups (Fig. 1). High initial concentrations in non-surviving patients with low or moderate SOFA scores resulted in a high progression rate towards multiple organ failure $(N=6 ; 100.0 \%$ and $N=25 ; 52.1 \%)$, with similar results found in patients with increasing concentrations over the first $24 \mathrm{~h}$ (e.g. moderate SOFA population: $N=15 ; 57.8 \%)$. Conversely, mortality in patients with low MR-proADM concentrations was predominantly due to non-sepsis-related causes $(N=14$; $60.9 \%$ ), with a low subsequent progression rate towards sepsis-related multiple organ failure in the total patient population with continuously low concentrations over the first $24 \mathrm{~h}(N=3 ; 1.4 \%)$.

Results suggest that initially high or increasing MR-proADM concentrations may help to identify patients with a high risk of progression towards sepsis-related multiple organ failure. Elevated microcirculation dysfunction and endothelial permeability may therefore play a significant role in driving the development of further organ dysfunction, as described previously [5]. Further studies in larger patient populations are essential to confirm these hypotheses.

* Correspondence: gunnar.elke@uksh.de

${ }^{1}$ Department of Anaesthesiology and Intensive Care Medicine, University Medical Center Schleswig-Holstein, Campus Kiel, Arnold-Heller-Str. 3 Haus 12, 24105 Kiel, Germany

Full list of author information is available at the end of the article 


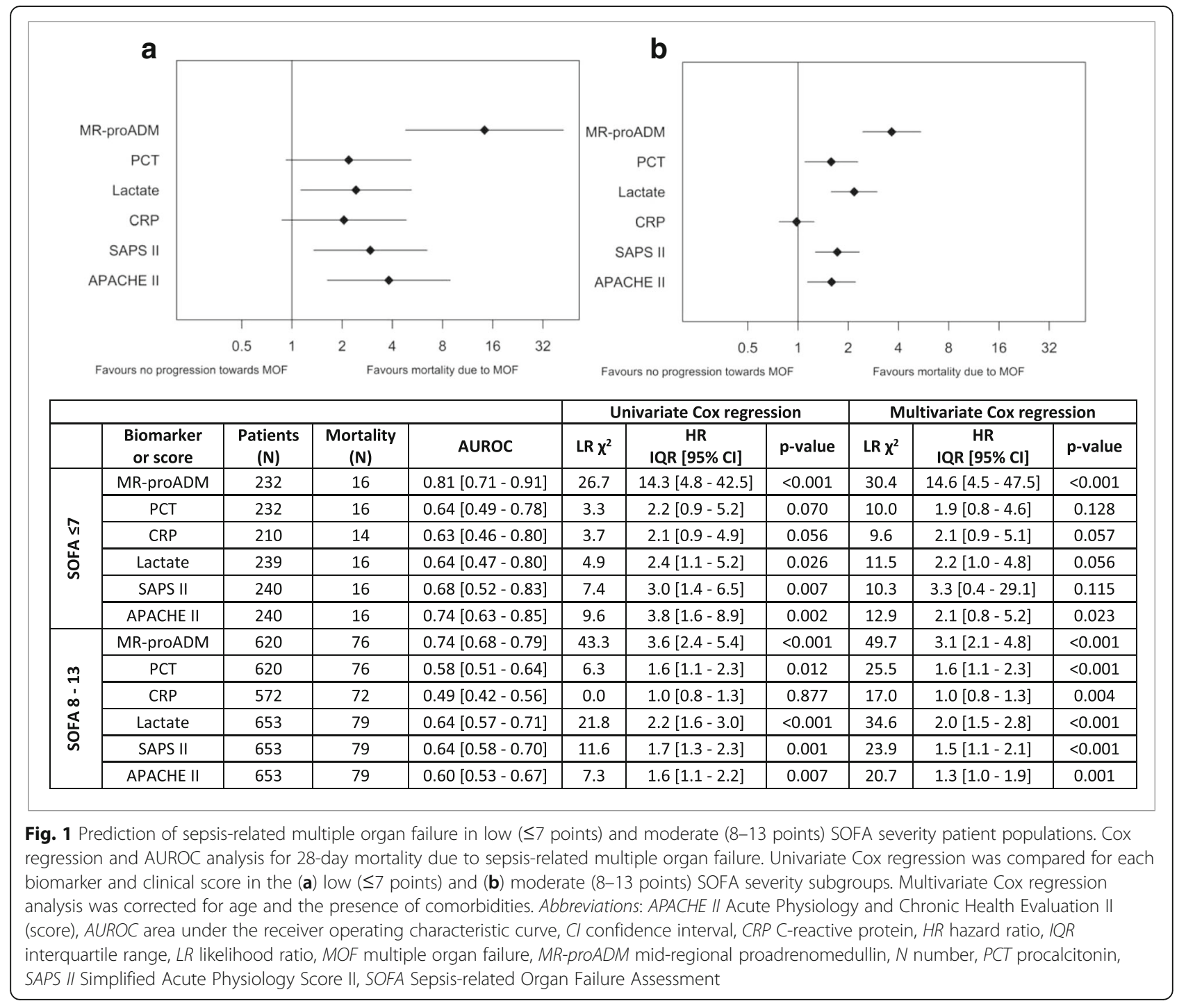

\section{Funding}

The SISPCT trial infrastructure was partially funded by grant $01 \mathrm{KI} 0106$ from the German Federal Ministry of Education and Research. Biosyn (Germany) and Thermo Fisher (Germany) provided study medication and financial support via unrestricted grants. For the purpose of this secondary analysis of the SISPCT trial, no specific funding was received. Neither for the original SISPCT trial nor for this secondary analysis did the funding organizations have any role in the design and conduct of the trial; collection, management, analysis, or interpretation of the data; preparation, review, or approval of the manuscript; or decision to submit the manuscript for publication.

\section{Availability of data and materials}

The datasets used or analyzed during the present study are available from the corresponding author on reasonable request.

\section{Authors' contributions}

GE was the primary author and editor of the manuscript. FB was the principal investigator for the SISPCT trial. All authors collected the study data and contributed to the evaluation and interpretation of data as well as writing and editing of the manuscript, performing the statistical analysis of data, and critically reviewing and approving the final manuscript.

\section{Ethics approval and consent to participate}

The study protocol of the Placebo Controlled Trial of Sodium Selenite and Procalcitonin Guided Antimicrobial Therapy in Severe Sepsis (SISPCT) was approved by the ethics board of Jena University Hospital (Internal File No. 2242-03/08). Written informed consent was obtained from all patients or their legal representatives.

\section{Consent for publication}

No individual participant data are reported that would require consent to publish from the participant (or legal parent or guardian for children).

\section{Competing interests}

FB reported receiving lecture honoraria from biosyn, Gilead, and CSL Behring and public funding for the SISPCT trial to his department by the German Federal Ministry of Education and Research as well as unrestricted research grants for the SISPCT trial by biosyn and Thermo Fisher Scientific. DCW is an employee of B.R.A.H.M.S GmbH. All other authors declared that they have no competing interests.

\section{Publisher's Note}

Springer Nature remains neutral with regard to jurisdictional claims in published maps and institutional affiliations. 


\section{Author details}

'Department of Anaesthesiology and Intensive Care Medicine, University Medical Center Schleswig-Holstein, Campus Kiel, Arnold-Heller-Str. 3 Haus 12, 24105 Kiel, Germany. ${ }^{2}$ Deparment of Anesthesiology and Intensive Care Medicine, Jena University Hospital, Am Klinikum 1, 07747 Jena, Germany. ${ }^{3}$ Center for Sepsis Control \& Care (CSCC), Jena University Hospital, Am Klinikum 1, 07747 Jena, Germany. ${ }^{4}$ B.R.A.H.M.S GmbH, Hennigsdorf, Neuendorfstr. 25, 16761 Hennigsdorf, Germany. ${ }^{5}$ Department of Anaesthesiology, Intensive Care Medicine and Pain Therapy, University Hospital Frankfurt, Theodor-Stern-Kai 7, 60590 Frankfurt am Main, Germany.

Received: 15 May 2018 Accepted: 24 May 2018

Published online: 05 June 2018

\section{References}

1. Elke G, Bloos F, Wilson DC, et al. The use of mid-regional proadrenomedullin to identify disease severity and treatment response to sepsis - a secondary analysis of a large randomised controlled trial. Crit Care. 2018:22:79.

2. Andaluz-Ojeda D, Nguyen HB, Meunier-Beillard N, et al. Superior accuracy of mid-regional proadrenomedullin for mortality prediction in sepsis with varying levels of illness severity. Ann Intensive Care. 2017;7:15.

3. Bernal-Morell E, García-Villalba E, Vera MDC, et al. Usefulness of midregional pro-adrenomedullin as a marker of organ damage and predictor of mortality in patients with sepsis. J Inf Secur. 2018;76:249-57.

4. Singer M, Deutschman CS, Seymour CW, et al. The third international consensus definitions for Sepsis and septic shock (Sepsis-3). JAMA. 2016;315:801-10.

5. Ince C, Mayeux PR, Nguyen T, et al. The endothelium in Sepsis. Shock. 2016; 45:259-70. 